case at the same time $(\mathrm{p}<0.05)$. Thus, the low level of intestinal sIgA complicates the course of disease.

\section{SOCIAL AND MEDICAL RISK INDICATORS IN ADHERENCE OF HIV/AIDS INFECTED CHILDREN BY VERTICAL TRANSMISSION}

doi:10.1136/archdischild-2012-302724.0946

R Hirsch. Universidad de Buenos Aires, Ciudad de Buenos Aires, Argentina

50 children were assisted at Hospital Muniz from 8/1/2010 to 2/28/2011: 11 boys (2/12), 11 girls (5/12), 28 teenagers: 9 males (12/17), 19 females (12/18). A social and medical score to value the adherence vulnerability was applied with these categories: annual internments, increase of viral load, decrease of CD4, clinical pathologies, relation between severity of pathology and internment length, lack of taking and/or delivering a medication, poor care support, lack of assistance to Short-Scheduled Hospitalization Program (SSHP). The results are: Low Score $(<3): \mathbf{2 4}$ patients. Slight Score $(3$ to $<6): 7$ patients. Moderate Score $(6$ to $<12): 8$ patients. High Score $(>12$ to $<24)$ : 11 patients. Nobody with low score presents social risks. Social risks were presented in: 5 slight score patients. 6 moderate score patients. 11 patients with high score. Suffered poor care support: 16 patients: 6 under $12(27.3 \%)$ and 10 adolescents, 7 women (36.8\%) and 3 males (33.4\%). Lack of taking and/or delivering a medication: 20 patients, 4 children under 12 years (18.2\%), 16 adolescents, 8 women (42.1\%) and 4 men (44.4\%) and lack of control to the SSHP, 15 patients: 4 children under 12 $(18.2 \%)$ and 15 adolescents, 8 women (42.1\%) and 3 males (33.3\%). Social and Medical risk in adherence indicators (shown at 2010 IAS Congress) adding the data provided by other disciplines managed to predict clinical evolution, to modify guidelines approach, to generate therapeutic strategies for monitoring at different ages, to follow ARV therapies in vertical transmission HIV/AIDS affected children.

\section{7 \\ SPREADING AND TREATMENT OF ACUTE MIDDLE OTITIS IN CHILDREN}

doi:10.1136/archdischild-2012-302724.0947

${ }^{1} \mathrm{~K}$ Gotsadze, ${ }^{2} \mathrm{~N}$ Adamia, ${ }^{3}$ C Chkhaidze, ${ }^{4} \mathrm{~K}$ Barabadze, ${ }^{3} \mathrm{E}$ Liluashvili, ${ }^{5} \mathrm{~L}$ Pachuashvili, ${ }^{6} \mathrm{~N}$ Nareklishvili. ${ }^{1} \mathrm{D}$. Tatishvili Medical Center; ${ }^{2}$ Department of General Pediatrics; ${ }^{3}$ Tbilisi State Medical University; ${ }^{4} / v a n e$ Javakhishvili State University; ${ }^{5}$ M. Iashvili Pediatric Clinic; ${ }^{6}$ M. Guramishvili Pediatric Clinic, Tbilisi, Georgia

Goal Development of optimal schemes against acute middle otitis in children through application of the mucoregulatory preparations in the complex antibiotic and basic therapy.

Materials and Methods Development of clinical course of acute middle otitis assessment criteria in children; Development of the optimal scheme against acute middle otitis according to disease severity in children; Treatment effectiveness evaluation for complicated acute middle otitis with mucoregulatory preparations in children; Evaluation of complex treatment effectiveness for complicated acute middle otitis in children. 936 patients from 3 months to 14 years were studied: 426 girls, 510 boys. For statistical processing SPSSv12 software was applied.

Obtained results Two groups of patients were studied: I: patients with complicated acute middle otitis (53-58.9\%); II: patients with acute middle otitis without complications (40.1\%); subjective criteria for eligibility: 1. disturbance, behavior changes (younger group), pain - 76\%; hearing discomfort $8 \%$; nasal signs $47 \%$; fever $-28.2 \%$. Otoscopic criteria: infiltration and hyperemia of tympanum $46.1 \%$; concaved or arched tympanum $29.3 \%$; changes of light reflex $24.6 \%$ $(p<0.001)$. Before treatment average values of mentioned symptoms were equal in both groups $(p=0.98)$ in case of acute middle year inflammation without complications mucoregulatory preparations (sinuforte) and sinupred were effective mono-therapeutic remedies. In case of complications mucoregulatory preparations are quite effective.

Conclusion Thus, effectiveness of monotherapy with the mucoregulatory preparations in case of acute middle otitis is similar to standard treatment and could be regarded as alternative remedy $p=0.01$. As for treatment of complicated disease combined treatment significantly reduces treatment duration $p<0.001$.

\section{OCCULT LUMBAR DERMOID CYST REVEALED BY RECURRENT KLEBSIELLA MENINGITIS}

doi:10.1136/archdischild-2012-302724.0948

P Flores, C Cristovao, L Tavora, AS Neto. Hospital Cuf Descobertas, Lisbon, Portugal

Introduction Lumbar dermoid cysts and other occult dysraphisms are sometimes difficult to diagnose. These anomalies are occasionally detected after a nervous central system infection.

Case Report Previously healthy 10 month-old child, admitted after a first febrile generalized seizure with nuchal rigidity and bulging fontanel. A very small blind lumbar dimple above the $5^{\text {th }}$ lumbar vertebra was noticed. Cerebrospinal fluid (CSF) was consistent with bacterial meningitis and penicillin susceptible Klebliella spp was identified. Brain CT-scan was normal and CSF was sterile after 4 days of ceftriaxone. She completed 21 days of therapy, with clinical improvement. One week after, she was readmitted with fever, lethargy and opisthotonus. Klebsiella oxytoca meningitis was diagnosed and treated for 3 weeks with ceftriaxone and gentamicin, according to antibiotic susceptibility test. Brain and spine magnetic resonance imaging showed a L4-L5 lumbo-sacral intrarachidian dermoid cyst with a fistulous path to skin surface. Surgical closure of fistula was performed on day 23 of therapy. This child is currently under antibiotic chemoprophylaxis with amoxicillin/clavulanate, awaiting removal of dermoid cyst under optimal sterile conditions. Neurological exam and motor development have been normal.

Conclusions A strong clinical suspicion is necessary in order to diagnose occult dysraphism and spinal midline cysts, before complications occur. A careful examination of the midline is warranted in all infants, paying special attention to skin pits outside coccigeal area, even if apparently blind. This diagnosis should also be considered in cases of recurrent or unusual bacterial associated central nervous system infections.

\section{MANAGEMENT OF PERI-ORBITAL/ORBITAL CELLULITIS IN CHILDRENACROSS TWO DISTRICT GENERAL HOSPITALS}

doi:10.1136/archdischild-2012-302724.0949

A Kage. Paediatrics, Leicester Royal Infirmary, Leicester, UK

Aim

1. To find out if there was uniformity in choice of antibiotics.

2. Are blood cultures, swabs and other blood tests helpful in management.

3. Look at involvement of other specialities in terms of followup and management.

Methodology Retrospective analysis of case notes with a diagnosis of preseptal/oribital cellulitis across 2 DGH. 15 such cases were recruited.

Results There was no uniformity in the antibiotics used. (5 different combinations were used).

Blood cultures were negative in 13 cases.

6 out of 15 had $C R P<15$.

Eye swab was positive in 3 cases.

Allied specialities were involved in 9 cases.

Only1 out of the total 15 cases developed an abscess and incidentally did not have anti-staphylococcal cover. 
Recommendations Education programme to raise awareness of complications of pre-septal/orbital cellulitis.

- Involve opthalmology and otolaryngology at the earliest.

- Twice daily assessment of colour vision, eye movements and pupil reflexes, for early identification of complications.

- Ensure adequate analgesia.

- Re-audit, preferably with the use of one antibiotic combination, also looking at indications for changing from IV to oral along with total duration.

\section{C-REACTIVE PROTEIN, LEUKOCYTES AND ESR AS IDENTIFIERS OF INVASIVE BACTERIAL INFECTIONS}

doi:10.1136/archdischild-2012-302724.0950

${ }^{1} \mathrm{C}$ Zavarache, 1,20 Falup-Pecurariu. 'Department of Pediatrics, University Children's Hospital; '2Department of Pediatrics, Faculty of Medicine, Transilvania University, Brasov, Romania

Background C-reactive protein (CRP), erythrocyte sedimentation rate (ESR) and leukocyte count are used in several studies, for evaluation of invasive bacterial infections.

Aim Aim of the study to evaluate the usefulness of leukocytes, CRP and ESR as identifiers of invasive bacterial infections at children.

Methods We have prospectively evaluated 705 patients divided into two groups: 1) group A with 110 patients having invasive bacterial infections and 2) group $B$ with localized bacterial infections comprised of 595 patients.

Results Mean values for leucocytes was $12536 \pm 6642 / \mathrm{mm}^{3}$ for invasive bacterial infections versus $11263 \pm 5285 / \mathrm{mm}^{3}$ for localized bacterial infections $(p<0.05)$.

Mean ESR for invasive infections was $31,13 \pm 29,11 \mathrm{~mm} / \mathrm{hr}$ versus $25,94 \pm 22,93 \mathrm{~mm} / \mathrm{hr}$ for localized infections ( $<<0.08$ ). Mean CRP for invasive infections was $42,57 \pm 25,45 \mathrm{mg} / \mathrm{dl}$ versus $24.10 \pm 13.58 \mathrm{mg} / \mathrm{dl}(\mathrm{p}<0.04)$ for localized bacterial one.

Conclusion This study suggests that CRP, ESR and leucocytes are good indicators in diagnose of invasive bacterial infections.

\section{RECURRENT PRIAPISM PRESENTING WITH A RARE CAUSE IN AN INFANT}

doi:10.1136/archdischild-2012-302724.0951

C Warren, T Fontaine, S Bedwani. Child Health Department, Royal Cornwall Hospital, Truro, UK

Discitis in children is an important infective cause of morbidity and often has an insidious presentation, which creates difficulty in making a prompt diagnosis and instigating appropriate treatment.

Priapism in the paediatric population is a rare urological emergency. It is most commonly associated with sickle cell disease and leukaemia, causing a vaso-occlusive priapism, and rarely with spinal shock. To the authors' knowledge, priapism secondary to discitis has not previously been described in the literature.

We present a case of a 12 month old Caucasian child presenting with a three-week history of regular episodes of seemingly painful, persistent erections. He was noted to have a reluctance to sit or walk. Clinical examination was unremarkable other than priapic episodes. Neurological and haematological causes were considered but initial investigations were normal.

An MRI of his spine, performed due to a later rise in erythrocyte sedimentation rate and a clinical deterioration with complete refusal to mobilise, demonstrated inflammatory changes at the L3/ L4 level with a seven-millimetre collection, thus indicating discitis. $\mathrm{He}$ was treated with intravenous antibiotics and after ten days made a full recovery with complete resolution of his symptoms.

This is the first reported case of discitis presenting as recurrent priapism in an infant. We feel that this case highlights the need to consider infective causes in children presenting with priapism, so that diagnosis and treatment of a significant underlying cause is not delayed. Early spinal imaging may be worthwhile in cases of unexplained priapism, particularly when haematological causes have been excluded.

\section{ENCEPHALITIS DUE INFLUENZA A (H1N1) IN A SEVEN YEAR-OLD GIRL WITH GOOD RESPONSE TO OSELTAMIVIR}

doi:10.1136/archdischild-2012-302724.0952

'F Abbasi, ${ }^{2} \mathrm{D}$ Yadegarynia, ${ }^{3} \mathrm{~S}$ Korooni, ${ }^{2} \mathrm{M}$ Razavi. ${ }^{1}$ Bushehr University of Medical Sciences, Bushehr; IInfectious Diseases and Tropical Medicine Research Center, Shaheed Beheshti Medical University, Tehran; ${ }^{3}$ Shiraz University of Medical Sciences, Shiraz, Iran

Background and Aims Human infection with the novel H1N1 influenza virus was first reported in April 2009. Novel Influenza A (H1N1) virus produces higher mortality in young people. Different clinical manifestation of Influenza A (H1N1) has been reported. We present encephalitis due influenza A (H1N1) with good response to oseltamivir

Patient The patient was a seven year-old girl presented with mood change and gait ataxia from 5 days before admission. She also had fever, delusion, and lethargy. She had history of common cold several days before admission. She was treated with acyclovir with impression of encephalitis without improvement. In physical examination $(\mathrm{P} / \mathrm{E})$ she was febrile, there was no nuchal rigidity. $\mathrm{P} / \mathrm{E}$ of chest, abdomen and extremities were normal. Lumbar puncture was performed. Cerebrospinal fluid (CSF) was normal. CSF culture showed no growth after 48h. CBC, FBS, BUN, Cr, Na, K, ALT, AST, CRP and procalcitonin were all normal. HSV PCR was negative. Elecroencephalography (EEG) was performed that suggested encephalitis. Brain MRI was normal. Throat culture was obtained for the diagnosis of influenza A (H1N1) that was positive. The patient was treated with oseltamivir. The patient recovered after treatment and tests for equilibrium became normal.

Conclusions Encephalitis due to influenza A (H1N1) should be considered in every patient with signs and symptoms of encephalitis during influenza $\mathrm{A}(\mathrm{H} 1 \mathrm{~N} 1)$ pandemy.

\section{MILIARY TUBERCULOSIS IN AN IMMUNOCOMPETENT CHILD}

doi:10.1136/archdischild-2012-302724.0953

'CSG Garcez, 'S Carvalho, 'S Martins, ${ }^{1} T$ Pontes, ${ }^{1} \mathrm{~A}$ Antunes, ${ }^{1} \mathrm{~A}$ Gonçalves, ${ }^{1} \mathrm{C}$ Moreira, 1,2H Antunes. 'Pediatrics, Braga Hospital, Braga; 'Life and Health Sciences Research Institute, School of Health Sciences, University of Minho ICVS/3B's - PT Government Associate Laboratory, Braga/Guimarães, Portugal

Background Miliary tuberculosis (MT) refers to clinical disease resulting from lymphohematogenous dissemination of Mycobacterium tuberculosis. It affects primarily younger and immunocompromised children. Clinical presentation is highly variable and multiorgan involvement is common.

Clinical Case A nine-year Gypsy girl with a mild development delay and a history of epilepsy was presented to our hospital with a 19-day history of fever. She had completed two antibiotic treatments for suspected tonsillitis. Two years before she had had meningococcal meningitis. Family history was irrelevant. On admission, physical examination was normal and it was performed a chest radiography which revealed diffuse, bilateral, small lung nodules (Panel A). Ophthalmology diagnosed tubercular choroiditis in the right eye. An acute MT diagnosis was made. Lumbar puncture was normal and cranial magnetic resonance imaging showed several small nodular lesions - tuberculomas. Mantoux test: $14 \mathrm{~mm}$. In gastric aspirate cultures grew Mycobacterium tuberculosis without antibiotic resistance. Immune deficiency was excluded. She received 